aged 9 to 14 . Of the London children 123 were boys aged 9 to 14 living in a camp school.

The tests began in Nov./Dec., 1941, and ended in July/Aug., 1942. The school-children were examined before, during, and after the period of feeding. They were divided at random into two equal groups according to school, age, and sex ; one group received a vitamin capsule every school day, and the other a capsule containing a similar quantity of arachis oil, which is practically devoid of vitamins. Records were kept of all illnesses, of their nature and duration. The medical examination included examination of the teeth and gums by dentists-in London by T. S. Rodgers.

The factory tests were conducted at the zinc-smelting works at $A$ and $L$, where the labour is extremely strenuous and exhausting. The men work in gangs of five or six, and the average output of zinc for each gang is weighed daily. In factory A 12, and in factory L 24, gangs of workers were divided at random into a vitamin and a control group. Each man received an appropriate capsule six days a week. The naked weight was taken monthly before the men began work. Haemoglobin and blood pressure were also measured at regular intervals, and the output of zinc and all absences due to illness were recorded.

The findings for the children were similar in all four places. During the period of observation the vitamins had no statistically significant effect on the rate of growth, nutritional status, muscular strength, condition of the teeth and gums, or absence from school on account of illness. Muscular strength was measured in London and Glossop by a dynamometer like that described by Hill, Magee, and Major (Lancet, 1937, 2, 441); it was not done in Ipswich. In Glossop alone a special test of endurance by hanging on a bar was done. Both the controls and the vitamin children improved in endurance, and the improvement shown by the vitamin children was greater than that shown by the controls. In view of the relatively small number of Glossop children tested, we feel that this particular finding should be strictly limited in its application to that group of children. Similar findings would have to be obtained in other areas before any wider application of the Glossop results could be regarded as justifiable. Feeding tests with this object were started on several thousand children three months after the conclusion of the above tests, and are still in progress.

In the factory tests on 214 adult men, the results showed that the vitamin capsules had no significant effects on weight, haemoglobin, blood pressure, absence from illness, or output of material. The conditions of the test in the factories, however, were such that only relatively large differences in output would have been revealed.

Many people assisted us in conducting these feeding tests and analysing the results, but lack of space forbids individual acknowledgment of their help. A mass expression of thanks to all who co-operated is all we can make in this summary.

\section{UMBILICAL HERNIA IN CHILDREN} WITH SPECIAL REFERENCE TO INJECTION TREATMENT

\section{BY}

M. J. BENNETT-JONES, Ch.M., F.R.C.S.

Honorary Surgeon, Bootle General Hospital and St. Helens Hospital; Visiting Surgeon, Whiston County Hospital

Umbilical hernia is classified into three types-congenital, infantile, and adult. There is no disagreement as to the treatment of the extremely rare congenital type, or exomphalos: all are agreed that it is only possible to save the infant from peritonitis by operating within a short period after birth. I have successfully operated on an infant within four hours of birth for an extensive exomphalos containing much large and small intestine: the defect in the abdominal wall was closed by the Mayo method, and it was surprising how the scar later contracted down and almost resembled a normal umbilical cicatrix.

The infantile and adult types are both of acquired origin, but they are regarded as being quite separate diseases. The general impression is that umbilical hernia rarely occurs in adolescence and early adult life. It is true that surgery is seldom requested for umbilical herniae at this period of life, because, if present, they are small and do not bother the patient much. Few mothers will fail to seek medical attention for a large umbilical hernia in a child. The adult patients one has to deal with are more commonly females than males, and the preponderance of females appears proportional to the greater incidence of adiposity in the female sex. Although the patients are over 40 years of age they usually state that they know the hernia has been present many years; but the onset is always vague and insidious. It is therefore impossible to establish any clear relation between the presence of an umbilical hernia in childhood and one in. later life.

\section{Clinical Features of Infantile Umbilical Hernia}

The mother nearly always states that the hernia has dated from soon after birth, but occasionally a latent interval up to four months has been noticed. There is no history of any gross sepsis of the umbilical stump. The infant, either male or female, is often poorly developed, and is more likely to be suffering from rickets than the very rare condition of cretinism. It is important to keep in mind the possibility of increased intra-abdominal pressure due to ascites or intestinal obstruction, etc. The majority, however, have no obvious predisposing cause, but sometimes inguinal hernia is also present: adiposity apparently is not associated with infantile umbilical hernia. The infants are presented at a surgical outpatient department from 8 weeks old upwards, but the majority are about 18 months old when they arrive, having failed to respond to treatment by strapping.

\section{Treatment}

Paterson and Gray (Barrington-Ward, 1937) found that out of 214 cases at the Hospital for Sick Children, Great Ormond Street, 101 disappeared without operation, but all writers say that the longer the hernia has been present and the larger the neck, the less likely will it be curod without operation. Few spontaneous cures occur after the age of 3 with support. As spontaneous cure with support occurs in nearly half the cases I thought injections would increase the proportion of, and hasten, cure in selected cases with small necks. My results seem to show that injections can cure umbilical hernia in such cases. The advantage of the treatment is that it may avoid operation and admission to hospital, and can therefore be advised for debilitated infants and children with small herniae. I think the main disadvantage is that the temperament of young children is rather unsuited to repeated injections if one is insufficient. There appears to be no risk of injecting into the peritoneal cavity.

Injection treatment of inguinal hernia was first practised by George Heaton (1877) of Boston, Mass., about a hundred years ago, but was later perfected by Ignatz Mayer of Detroit. Delisle Gray (1932), who advocated Mayer's technique, brought the method and the results before the medical profession in England. So far as I can.ascertain from literature and hearsay evidence there have been few cases of umbilical hernia treated in this way. Burdick and Coley (1937), out of a total of 92 cases, treated only one umbilical hernia by injection, and the result of this was not stated. Wyss (1929), Larson (1934), and Quillin (1934) reported favourably on the result of injecting umbilical herniae, but they were all mainly concerned with adult inguinal hernia. Bratrud (1937) illustrated his technique of injections for umbilical hernia, and stated that by this method they were more favourable than even in indirect inguinal hernia. Personally, I have not yet tried injections for inguinal hernia because the many attendances for injections at an out-patient department would entail as much lost work as with operative treatment, and if the patient was not cured at the end of the time both patient and employer would be dissatisfied. With regard to inguinal hernia in children, the operative treatment is so safe, with recurrence and sepsis almost unknown, that I have not yet been tempted to try injection treatment. There is no doubt that even inguinal hernia can sometimes be cured by a truss in childhood-e.g., the famous case of Sir Astley Cooper (Murray, 1910), which was verified at his necropsy, when only a minute sinus canal was found extending to the tunica vaginalis. In spite of this, operative treatment is indicated because of the rarity of spontaneous cure.

It is the accepted view that umbilical hernia in children is nearly always curable with a support, but the cases seen 
in hospital do not seem to respond to strapping. The cases of umbilical hernia I selected for injection had necks the smallest diameter of which was one-third of an inch or less. Most of the cases had been sent to me because strapping had failed.

\section{Technique of Injection Treatment}

An anaesthetic is not essential for young infants; a little ethyl chloride anaesthesia, however, is very helpful, because voluntary movements are controlled, but the sac still remains distended, so that it can be avoided. I have used up to $4.5 \mathrm{c.cm}$. of $5 \%$ phenol in almond oil at one injection. The solution is injected into the subcutaneous tissue as near the neck as possible without risk of puncture of the peritoneum. At a subsequent injection, it this is required, it is much harder to inject the solution. After injection it is essential to keep the hernia reduced. A small round pad of gauze is held in position by elastoplast applied right round the body like a belt at the level of the umbilicus. This support should be continued after the injections have ceased, because the scar tissue produced by the sclerosing fluid becomes more strain-resistant as time progresses. Animal experiments (Crohn, 1937) have shown that the phenol solution produces tiny areas of necrosis, which are later followed by more intense fibrous reaction than that seen when other solutions have been tried.

I would like to emphasize the method of applying elastoplast as a complete belt. Beadnell (1942) has suggested applying elastoplast to one half of the abdomen as far as the midline while the nurse pushes the other half of the rectus muscle towards the middle. Beadnell admits that his method produces a fold of skin in the midline, so there will be no effective antero-posterior support and very little pull on the two rectus muscles when the child cries or strains. The belt method of application keeps the hernia effectively reduced without any skin-chafing, and it will give much more support to the hernia owing to fixation from the lumbar spine. Mothers of babies with umbilical hernia nearly always state that they have noticed that the baby has cried less than before, and the belt method has certainly not reduced the baby's appetite or increased its liability to vomit. I strongly recommend this method of support, even for the youngest babies, as soon as umbilical hernia is noticed. The ordinary binder slips either up or down and it has to be removed daily for bathing, so the hernia is not kept permanently reduced.

\section{Results of Injection Treatment}

With ages ranging between nearly 3 months and 5 years, 42 out of a total of 62 cases of infantile umbilical hernia were selected for injection treatment and 63 injections were given. 26 cases needed only one injection; 11 cases had two injections, but two of these were submitted to operation later because the hernia showed insufficient diminution in size $; 4$ cases had three injections, and only 1 case had four injections, but this resulted in a cure. Out of the 42 cases selected for this treatment 31 were apparently completely cured when seen later, excluding the 2 cases cured by operation after unsuccessful injections ; 2 cases were untraced, but it was not certain that the remaining 7 were finally cured, although when last seen they were responding well and there was very little umbilical bulge.

\section{Subcutaneous Ligature}

This minor operative procedure is recommended by Fraser (1926) for small umbilical herniae, and especially if they occur in slightly debilitated children who might not stand the radical procedure. The same author states that he has had no recurrence in hundreds of cases. Others, including myself, have not found this method so certain, because there has been a recurrence after an interval. It is probable that I would have found it more successful if I had combined subcutaneous ligature with a post-operative belt support.

\section{Radical Cure}

Most writers state that the radical method is the only certain way of curing umbilical hernia if the patient is over 3 . It is required, however, for large herniae below that age. BarringtonWard (1937) does not advise the Mayo type of repair, because there is no wide separation of the fascia, and yet he states that the recti are separated. It is curious that $I$ cannot find any textbook which mentions that the hole in the fascia is more often elliptical, with the long axis transverse; in fact, it can often be described as a transverse slit. Surely this shows. that the Mayo type of closure of the fascia is better, just as it has proved better in adults. In children it is unnecessary to excise the umbilicus with an elliptical area of skin as in the Mayo operation for adults, because the hernia is reducible.

After opening the sac at its neck it is often possible to sew up the peritoneum separately. The aponeurotic opening is then closed by mattress sutures with overlap. The most difficult part of the operation is dissecting the fundus of the sac off the deep aspect of the umbilicus without buttonholing it or leaving a portion of the sac behind. My impression is that it is better to buttonhole the skin than to leave any endothelial lining, which predisposes to haematoma formation and sepsis in spite of drainage. Recently I have not drained the wound but have applied elastoplast as a belt after the operation. This procedure obliterates any dead space, and appears to have reduced the incidence of slight wound sepsis. Because of the danger of collapse of the lung Fraser does not recommend this operation before the age of 3: Apart from local sepsis, which delays convalescence, I have had no complications, although I will undertake operation in much younger patients if the hernia is large. I have performed the radical operation in 30 children, with two recurrent cases, during the same period as the cases that were selected for injection treatment.

Conclusions
Infantile umbilical hernia should be treated as early as possible with elastoplast applied as a belt with a small pad. After the age of 3 months the combination of injection and support will give a large percentage of cures even if strapping has failed in about two-thirds of the cases sent to the surgeon. Ambulatory treatment is desirable if possible in children, because of the frequent shortage of children's beds through infectious diseases and because of the risk of cross-infection. The injection treatment appears safe if done under short general anaesthesia.

REFERENCES

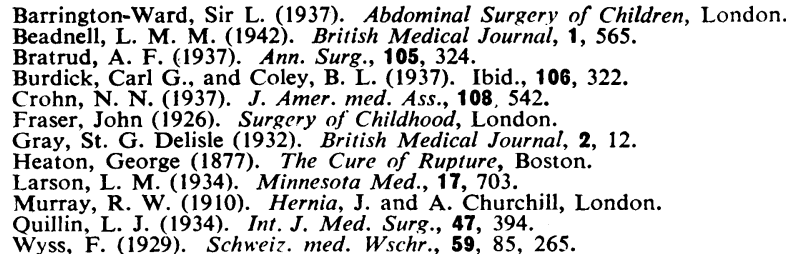

\section{Medical Memoranda}

\section{Dienoestrol for Menopausal Symptoms}

The chemical formula and physical properties of dienoestrol $\left(\gamma \delta\right.$-di- $p$-hydroxyphenyl- $\Delta^{\beta \delta}$-hexadiene) were recorded by Dodds, Golberg, Lawson, and Robinson (1938), and Campbell, Dodds, Lawson, and Noble (1939). Zuckerman (unpublished communication), working on monkeys, found that dienoestrol when given by injection was much less potent than the naturally cccurring oestrogens. Emmens (1938) found that when this compound was given to mice by mouth it had the greatest activity, relative to subcutaneous dose, of any oestrogen examined up to that time.

A clinical trial with dienoestrol was carried out by me (Barnes, 1942). The compound was used for the inhibition of lactation, and its effect for this purpose was compared with that of stilboestrol and hexoestroi. The results showed that when given by mouth dienoestrol is a potent oestrogenic substance, and inhibition of lactation was obtained with one-tenth of the dose required in the cases of stilboestrol and hexoestrol. It was clear that further clinical trials were desirable to establish the value of dienoestrol as an oestrogenic agent, and it was therefore decided to try its effect in relieving menopausal symptoms.

Hawkinson (1938) as a result of the study of 1,000 women who were undergoing a natural or artificially induced menopause found that $75 \%$ suffered from distressing symptoms, but that considerable relief could be given by the administration of naturally occurring oestrogens. This has been the experience of many workers, and it has been found that the synthetic oestrogens are equally successful in relieving the symptoms. 